\title{
Status of insecticide resistance in malaria vectors in Kwale County, Coastal Kenya
}

\author{
Caroline W. Kiuru ${ }^{1,2}$, Florence Awino Oyieke1, Wolfgang Richard Mukabana ${ }^{1,3}$, Joseph Mwangangi ${ }^{2,4}$, \\ Luna Kamau ${ }^{5}$ and Damaris Muhia-Matoke ${ }^{5^{*}}$
}

\begin{abstract}
Background: The strategy for malaria vector control in the context of reducing malaria morbidity and mortality has been the scale-up of long-lasting insecticidal nets to universal coverage and indoor residual spraying. This has led to significant decline in malaria transmission. However, these vector control strategies rely on insecticides which are threatened by insecticide resistance. In this study the status of pyrethroid resistance in malaria vectors and it's implication in malaria transmission at the Kenyan Coast was investigated.

Results: Using World Health Organization diagnostic bioassay, levels of phenotypic resistance to permethrin and deltamethrin was determined. Anopheles arabiensis showed high resistance to pyrethroids while Anopheles gambiae sensu stricto (s.s.) and Anopheles funestus showed low resistance and susceptibility, respectively. Anopheles gambiae sensu lato (s.l.) mosquitoes were further genotyped for L1014S and L1014F kdr mutation by real time PCR. An allele frequency of 1.33\% for L1014S with no L1014F was detected. To evaluate the implication of pyrethroid resistance on malaria transmission, Plasmodium falciparum infection rates in field collected adult mosquitoes was determined using enzyme linked immunosorbent assay and further, the behaviour of the vectors was assessed by comparing indoor and outdoor proportions of mosquitoes collected. Sporozoite infection rate was observed at 4.94 and 2.60\% in An. funestus s.l. and An. gambiae s.l., respectively. A higher density of malaria vectors was collected outdoor and this also corresponded with high Plasmodium infection rates outdoor.

Conclusions: This study showed phenotypic resistance to pyrethroids and low frequency of L1014S kdr mutation in An. gambiae s.l. The occurrence of phenotypic resistance with low levels of $k d r$ frequencies highlights the need to investigate other mechanisms of resistance. Despite being susceptible to pyrethroids An. funestus s.l. could be driving malaria infections in the area.
\end{abstract}

Keywords: Malaria vectors, Insecticide resistance, $k d r$

\section{Background}

In the last decade, there have been global efforts to reduce malaria morbidity and mortality through different programmes [1]. In Kenya, the main strategies employed by the National Malaria Control Programme have been: (1) scaling up of vector control interventions; (2) timely diagnosis and effective treatment using artemisininbased combination therapy (ACT); and (3) intermittent preventive treatment for pregnant women (IPTP) [2]. For

\footnotetext{
*Correspondence: dmatoke@kemri.org

${ }^{5}$ KEMRI-Centre for Biotechnology Research and Development, Nairobi, Kenya

Full list of author information is available at the end of the article
}

vector control, the World Health Organization (WHO) recommends long-lasting insecticidal nets (LLINs), indoor residual spraying (IRS) and larval source management $[3,4]$. The most common and widespread of these methods is the use of IRS and LLINs, with LLINs being more dominant due to ease of distribution and low cost associated with their roll out [5].

Pyrethroids remain the only class of insecticides recommended for the treatment of LLINs. This arises from their low toxicity to humans and their mode of action which entails rapid and persistent effects against mosquitoes [6]. Until an alternative is obtained, vector control using LLINs remains heavily dependent on pyrethroids. 
In Kenya, a mass bed net distribution campaign in malaria endemic areas was held in 2006, where LLINs given to pregnant women and children below the age of 5 years attained a coverage of $60 \%$ [7]. In 2012, a near universal coverage was attained with another mass distribution of LLINs, where the goal of one net in every two people in a household was reached [8]. This increased ITN coverage coupled to the other malaria control strategies has seen reduction in malaria transmission in many localities. Unfortunately, the sustainability of vector control using LLINs remains questionable due to insecticide resistance. Already the last decade has seen increased reports of resistance to pyrethroids in at least 27 countries in Africa [9]. In Kenya, resistance has been reported in Western Kenya, with no report on the current state of insecticide resistance in the Kenyan Coast despite intensified vector control in the area [10-12].

The main malaria vectors in the Kenyan Coast are; Anopheles funestus sensu lato (s.l.) and Anopheles gambiae s.l., both of which are complex species [13]. Members of both complexes exhibit variation in their biology making it difficult to have a universal control. With LLINs targeting malaria vectors biting indoors, the occurrence of resistance could greatly impact malaria transmission dynamics. Changes in species composition, as well as, changes in their role in transmission have been reported in relation to increased bed-net use and coverage $[14,15]$. With the increased use of pyrethroids for LLINs, IRS and in agriculture mosquitoes are subjected to insecticide pressure thereby, increasing their probability of resistance [16].

In the light of the increased bed-net coverage and the likelihood of this serving as a potential selective pressure for malaria vectors, this study sort to assess the level of phenotypic and genotypic resistance in Kwale County in the Kenyan Coast. In an effort to understand the implication of resistance on malaria transmission, species composition, sporozoite infection and indoor/outdoor proportions of malaria vectors was assessed.

\section{Methods}

\section{Study area}

The study was conducted in South Coast Kenya in Kwale County. The study area has been previously described [17]. Anopheles funestus s.l. and An. gambiae s.l. are the main malaria vectors in the area $[18,19]$. They occur all year round, with peak season during the rainy season [18]. In the area, $50 \%$ of households have universal ITN coverage ( $\leq 2$ persons per ITN). Generally, for the Kenyan Coast an increase in malaria prevalence from 4 to $8 \%$ since 2010 has been reported. Sampling was done in two villages; Marigiza (Latitude -4.443036 , Longitude 39.461887) and Kidomaya (Latitude - 4.578639,
Longitude 39.157574) which are about $50 \mathrm{~km}$ apart representing the Coastal plain and Coastal estuarine habitats, respectively. The history of bed net use in Kidomaya dates back to 1998 where all households were provided with insecticide-treated nets (ITNs) as part of a clinical trial [20]. After this, the two villages have received parallel distribution of LLINs through the National Malaria Control Programme by mass distribution campaigns held in 2006 and 2012.

\section{Mosquito collection and rearing}

Mosquitoes were collected in July and August 2015, which corresponds to the dry season. Adult mosquitoes were collected using light traps, which have been reported to be efficient in collecting host-seeking mosquitoes [19]. Additionally, Light traps were supplemented with Prokopack aspirator (John W. Hock Co., Gainesville, FL, USA) to capture indoor resting mosquitoes.

In each trapping night, light traps were set up both inside and outside three randomly selected houses in each village between 1800 and $1700 \mathrm{~h}$. Indoor traps were set up at the foot side of the bed $1 \mathrm{~m}$ off the ground and approximately $1.5 \mathrm{~m}$ from the place of sleep [21]. The outdoor traps were placed at least $5 \mathrm{~m}$ from houses containing the indoor light traps. The traps were removed the following morning between 0600 and $0700 \mathrm{~h}$. To boost the number of blood-fed mosquitoes collected, aspiration was done in the same and nearby houses within $500 \mathrm{~m}$ radius in the morning between 0700 and $0900 \mathrm{~h}$ using Prokopack aspirator with a view of maximum sampling. Live mosquitoes collected by light traps and aspirators were transferred into paper cups, provided with $6 \%$ sucrose and stored in a cool box for transportation to Msambweni Hospital Research laboratory for further processing.

Adult mosquitoes collected were sorted according to their sex and physiological status i.e. as gravid, half gravid, blood fed and unfed. Live mosquitoes that were gravid, half gravid and blood-fed were kept in paper cups in the insectary. They were provided with $6 \%$ sucrose, when fully gravid they were transferred to individual egg laying tubes. The egg laying tubes were perforated $1.5 \mathrm{ml}$ eppendorf tubes lined with a moistened strip of filter paper [22]. Eggs from individual females were reared in separate trays to obtain iso-female families.

Larvae collection was done in all identified water bodies in each village. Approximately five larval habitats were sampled in each village per week. Initially, larvae were collected using the standard dipping method whereby, ten dips were made per potential larval habitat using a standard $350 \mathrm{ml}$ dipper [23]. As the densities of Anopheles larvae and the number of habitats were low, the number of dips was increased and larvae were collected 
exhaustively from the habitats. This adjustment was in accordance to WHO guidelines for monitoring insecticide resistance, that larval collections be made from a number of different breeding habitats to avoid collecting larvae from single egg batches [24]. Collected larvae were transported in Whirl-Pak ${ }^{\circledR}$ bags to the laboratory where larvae from the same village were pooled together and sorted by their instar stages. Larvae were maintained using Tetramin ${ }^{\circledR}$ baby fish food.

\section{Insecticide susceptibility bioassays}

Female, F0 adults reared from larval collections were used for the bioassay. This is because no $\mathrm{F} 1$ adults were obtained from field collected adults due to high larval mortalities. Non-blood fed 3-5 days old female adults were exposed to $0.75 \%$ permethrin and $0.05 \%$ deltamethrin or control papers impregnated with silicone oil at temperatures of $25 \pm 2{ }^{\circ} \mathrm{C}$ and $70-80 \%$ relative humidity according to $\mathrm{WHO}$ insecticide susceptibility test guidelines [24]. Anopheles mosquitoes in batches of 18-25 were placed in holding tubes for $1 \mathrm{~h}$ after which any moribund mosquito was removed before being transferred to exposure tubes lined with insecticide or silicone oil impregnated papers. The tubes were held in vertical position and the knockdown rate recorded at intervals of $10,15,20,30,40,50$ and $60 \mathrm{~min}$. After $60 \mathrm{~min}$ the mosquitoes were transferred to holding tubes, maintained on $6 \%$ glucose and mortality rate determined $24 \mathrm{~h}$ post exposure. Laboratory reared An. gambiae sensu stricto (s.s.) Kisumu strain were exposed to each insecticide as positive control while $20-25$ field collected adults were exposed to control papers as negative control and used in correcting mortality rate using the Abbotts's formula [24]. Mortality rate was calculated by expressing the total number of dead mosquitoes from all four replicates for an individual insecticide as a percentage of the total exposed.

\section{Mosquito identification and sibling differentiation}

Filed collected adults and adults emerging from field collected larvae were identified morphologically to species level [25] and preserved on silica gel granules at room temperature. The field collected adults were later dissected into three portions; (1) head and thorax, (2) legs and wings, and (3) abdomen. For field collected adults, genomic DNA was extracted from the legs and wings. The other body sections were used for other analysis such as sporozoite ELISA analysis. For adults emerging from field collected larvae, genomic DNA was extracted from the whole body, and subjected to species ID and KDR analysis [26]. The DNA was used for sub-species identification for An. gambiae [27] and An. funestus [28] complexes by conventional polymerase chain reaction (PCR).
For the An. gambiae complex, further analysis to differentiate $\mathrm{M}$ and $\mathrm{S}$ molecular forms was not performed, as only the $\mathrm{S}$ form has been reported to be present in East Africa [29].

\section{Detection of $k d r$ mutations}

For the An. gambiae complex, the genomic DNA was further used to test for the presence of point mutations at the position 1014 of the voltage gated sodium channel by real time PCR, TaqMan probe based assay [30]. Both the leucine to phenylanine substitution ( $k d r$ west allele) and leucine to serine substitution ( $k d r$ east allele) were tested.

\section{Sporozoite analysis}

The heads and thoraces of individual anopheline females were tested for the presence of Plasmodium falciparum circumsporozoite antigen using sandwich enzyme-linked immunosorbent assay (ELISA) [31]. The infection rate was calculated as the proportion of infected mosquitoes.

\section{Data analysis}

Data was entered in Microsoft Excel 2010 and analysed using $\mathrm{R}$ software, version 3.3.2. Resistance was determined using the WHO classification of mortality rate where $98-100 \%$ mortality indicates susceptibility, 90-97\% suggests possible resistance for which further investigation is required while $<90 \%$ is considered resistance. Frequency counts for categorical data were compared using Pearsons's Chi square test performed at 0.05 level of significance.

\section{Results}

\section{Species composition}

A total of 1101 Anopheles adults and larvae were collected from the two villages, Marigiza (520) and Kiodomaya (581). Of these, $63.03 \%(n=694)$ were collected as adults while $36.97 \%(\mathrm{n}=407)$ were collected as larvae. From the adults collected, 154 were gravid, half-gravid or blood-fed, and were placed in oviposition tubes. The oviposited eggs hatched but did not survive past the 2nd larval instar. It is worth noting that only two blood-fed $A n$. gambiae s.l. were collected and only one laid eggs which hatched but also did not survive.

Overall, the proportion of An. funestus s.l. collected was higher (64.40\%) compared to An. gambiae s.l. (33.97\%) and other secondary malaria vectors $(1.63 \%)$ $\left(\mathrm{X}^{2}=650.72, \mathrm{df}=2, \mathrm{p}<0.001\right)$. The secondary malaria vectors collected include; Anopheles squamosus $(\mathrm{n}=7)$, Anopheles coustani $(\mathrm{n}=5)$, Anopheles pharoensis $(\mathrm{n}=5)$ and Anopheles pretoriensis $(\mathrm{n}=1)$ (Table 1). The proportion of An. gambiae s.l. collected in Kidomaya was significantly higher compared to Marigiza $\left(\chi^{2}=13.10, \mathrm{df}=1\right.$, $\mathrm{p}=0.003$ ) while no differences in An. funestus s.l. was 
observed between the two villages. Amongst the 374 An. gambiae s.l. collected, $88.24 \%$ were Anopheles arabiensis, $4.81 \%$ were $A n$. gambiae s.s. while $6.95 \%$ did not amplify (Table 1).

Out of 709 An. funestus s.l. collected, PCR species identification revealed that $76.02 \%$ were $A n$. funestus s.s., 3.53\% Anopheles leesoni, 2.96\% Anopheles parensis, 1.55\% Anopheles rivulorum, 0.71\% Anopheles vaneedeni and $0.85 \%$ hybrids, while $14.39 \%$ did not amplify. Hybrids

Table 1 Species composition of Anopheles mosquitoes collected in Marigiza and Kidomaya villages in Kwale County, Coastal Kenya

\begin{tabular}{|c|c|c|c|c|}
\hline Species & Sibling species ID & Kidomaya & Marigiza & Total \\
\hline \multirow[t]{4}{*}{ An. gambiae } & An. arabiensis & 183 & 147 & 330 \\
\hline & An. gambiae s.s. & 16 & 2 & 18 \\
\hline & Not amplified & 23 & 3 & 26 \\
\hline & Total & 222 & 152 & 374 \\
\hline \multirow[t]{8}{*}{ An. funestus } & An. funestus s.s. & 250 & 289 & 539 \\
\hline & An. leesoni & 24 & 1 & 25 \\
\hline & An.parensis & 13 & 8 & 21 \\
\hline & An. rivulorum & 2 & 9 & 11 \\
\hline & An. vaneedeni & 1 & 4 & 5 \\
\hline & Hybrids & 2 & 4 & 6 \\
\hline & Not amplified & 63 & 39 & 102 \\
\hline & Total & 355 & 354 & 709 \\
\hline An. coustani & & 3 & 2 & 5 \\
\hline An. pharoensis & & 0 & 5 & 5 \\
\hline An. squamosus & & 0 & 7 & 7 \\
\hline An. pretoriensis & & 1 & 0 & 1 \\
\hline
\end{tabular}

were identified based on production of two bands corresponding to two different sibling species after PCR amplification. The composition of the hybrids was: one $A n$. parensis/An. leesoni, one An. funestus/An. parensis and four $A n$. vaneedeni/An. parensis. However, the hybrids identified in this study will need to be analysed further as the occurrence of hybrids has been associated with sequence similarity between other species and members of $A n$. funestus complex in the internal transcribed spacer region 2 of the rDNA. The dominant An. funestus sibling species was $A n$. funestus s.s. in both villages.

\section{Outdoor and indoor collections}

Overall, no difference was observed between the total numbers of mosquitoes collected outdoor and indoor. In the interest of fairly comparing indoor and outdoor proportions we considered only mosquitoes collected by light traps for analysis in this section as aspirators were not used for outdoor collections. A higher number of mosquitoes was collected outdoor $(76.13 \%, \mathrm{n}=370)$ compared to indoor $(23.78 \%, \mathrm{n}=116)\left(x^{2}=132.75\right.$, $\mathrm{df}=1, \mathrm{p}<0.001)$. Only An. rivulorum had higher proportions indoor compared to outdoor (Table 2).

\section{Insecticide susceptibility bioassay}

A total of 407 F0 female adult mosquitoes aged 3-5 days old raised from larvae were used to test for susceptibility to deltamethrin and permethrin. From the 407, 72.24\% ( $\mathrm{n}=294$ ) were $A n$. gambiae s.1., 27.03\% ( $\mathrm{n}=110) A n$. funestus s.l. and $0.74 \%(\mathrm{n}=3)$ An. squamosus. Of these, 155 and 201 were exposed to deltamethrin and permethrin impregnated papers, respectively, while 51 were

Table 2 Total number and proportion of mosquitoes collected by light trap indoors and outdoors

\begin{tabular}{|c|c|c|c|c|}
\hline Species & Sibling species & Total & Indoor proportion (\%) & Outdoor proportion (\%) \\
\hline \multirow[t]{8}{*}{ An. funestus s.l. } & - & 397 & 24.69 & 75.31 \\
\hline & An. funestus s.s. & 280 & 22.14 & 77.86 \\
\hline & An. leesoni & 24 & 16.67 & 83.33 \\
\hline & An. parensis & 14 & 28.57 & 71.43 \\
\hline & An. rivulorum & 5 & 60 & 40 \\
\hline & An. vaneedeni & 3 & 66.67 & 33.33 \\
\hline & Hybrids & 3 & 0 & 100 \\
\hline & Not amplified & 68 & 33.82 & 66.18 \\
\hline \multirow[t]{4}{*}{ An. gambiae s.l. } & - & 74 & 22.97 & 77.03 \\
\hline & An. arabiensis & 55 & 23.64 & 76.36 \\
\hline & An. gambiae s.s. & 2 & 50 & 50 \\
\hline & Not amplified & 17 & 17.65 & 82.35 \\
\hline An. coustani & - & 5 & 0 & 100 \\
\hline An.pharoensis & - & 5 & 0 & 100 \\
\hline An. squamosus & - & 4 & 25 & 75 \\
\hline An. pretoriensis & - & 1 & 0 & 100 \\
\hline
\end{tabular}


Table 3 Mortality rate in female Anopheles mosquitoes exposed to deltamethrin and permethrin

\begin{tabular}{lllc}
\hline Insecticide & Species & Sibling species & Mortality \\
\hline Deltamethrin & An. funestus s.l. & - & $54(100)$ \\
& & An. funestus s.s. & $49(100)$ \\
& An. vaneendeni & $1(100)$ \\
& Not amplified & $4(100)$ \\
& An.gambiae s.l. & - & $101(62.38)$ \\
& & An. arabiensis & $95(61.05)$ \\
& & An. gambiae s.s. & $4(75)$ \\
& Not amplified & $2(100)$ \\
& & - & $41(100)$ \\
& An. funestus s.l. & An. funestus s.s. & $38(100)$ \\
& & Not amplified & $3(100)$ \\
& & - & $160(72.50)$ \\
& An. gambiae s.l. & An. arabiensis & $143(69.93)$ \\
& An. gambiae s.s. & $12(100)$ \\
& Not amplified & $5(80)$ \\
& & &
\end{tabular}

Number outside parenthesis is the total number exposed to insecticide impregnated papers. Number inside parenthesis is the mortality rate in $\%$

Table 4 Frequency of Knockdown resistance allele in relation to phenotypes determined by WHO susceptibility bioassay in Anopheles gambiae s.s.

\begin{tabular}{lrllll}
\hline Bioassay phenotype & $\mathbf{n}$ & \multicolumn{3}{l}{ L1014S kdr genotype } & F (kdr) \\
\cline { 3 - 5 } & & RR & RS & SS & \\
\hline Resistant & 78 & 0 & 1 & 77 & 0.0064 \\
Susceptible & 169 & 3 & 1 & 165 & 0.0207 \\
\hline
\end{tabular}

$\mathrm{R}$ represents the resistant allele, $\mathrm{S}$ represents the wild type/susceptible allele, $\mathrm{n}$ is the total number tested and $\mathrm{F}$ is the frequency of the $k d r$ allele. The resistant bioassay phenotype refers to mosquitoes that were alive $24 \mathrm{~h}$ post-exposure to either deltamethrin or permethrin while susceptible phenotype refers to those that were dead

exposed to the control papers impregnated with silicone oil. From the 356 exposed to insecticide treated papers, an overall mortality of $76.97 \%$ was observed with a mortality rate of 75.48 and $78.11 \%$ for deltamethrin and permethrin, respectively. The mortality rate for $A n$. gambiae s.s. Kisumu stain was $100 \%$ indicating full susceptibility to the insecticides and therefore confirming the effectiveness of the insecticide impregnated papers. No mortality was observed for the negative control with silicone oil impregnated papers, thus there was no need to correct for natural causes of mortality using the Abbott's formula.

All An. funestus s.l. tested showed $100 \%$ susceptibility to both deltamethrin $(\mathrm{n}=54)$ and permethrin $(\mathrm{n}=41)$ (Table 3). For An. gambiae s.l. overall mortality upon exposure to pyrethroids was $68.58 \%$ with mortality to deltamethrin and permethrin being 62.38\% $(\mathrm{n}=101)$ and $72.50 \%(\mathrm{n}=160)$, respectively both of which indicate resistance to pyrethroids. For the specific An. gambiae s.l. species, An. arabiensis exhibited an overall mortality to pyrethroids of $66.52 \%$ with a higher mortality to permethrin (69.93\%) compared to deltamethrin (61.05\%). Compared to An. arabiensis, An. gambiae s.s. showed a higher overall mortality to pyrethroids 92.86 with $100 \%$ mortality to permethrin and $75 \%$ mortality to deltamethrin. This indicates that An. gambiae s.s. are susceptible to permethrin and resistant to deltamethrin. As no differences in mortality rate was observed between the two villages, Kidomaya and Marigiza mortality rate data was analysed together for both villages (Table 3).

\section{Knockdown resistance mutations}

Three hundred $A n$. gambiae s.l. were genotyped for $k d r$ East (L1014S) and $k d r$-West (L1014F) mutations. Out of the 300 mosquitoes, 53 were from field collected adults while 247 were from the adults used for the bioassay, 79 of which had exhibited the resistance phenotype upon exposure to pyrethroid impregnated papers. Only L1014S mutation was detected in five An. gambiae s.s. 3 of which were homozygous while 2 were heterozygous for the L1014S allele. Of the 79 mosquitoes that had exhibited the resistance phenotype after exposure to pyrethroids only one An. gambiae s.s. showed genotypic resistance and was heterozygous for the L1014S allele (Table 4).

\section{Sporozoite infection rates}

Six hundred and fifty-nine mosquitoes were tested for the presence of $P$. falciparum parasites. Thirty tested positive giving an overall infection rate of $4.55 \%$. The infection rate was higher in An. funestus 4.94\% $(\mathrm{n}=567)$ compared to An. gambiae $2.60 \%(\mathrm{n}=77)$, these did not differ significantly $\left(x^{2}=0.8364, p=0.36\right)$. For secondary malaria vectors collected no sporozoite infection was detected. There was no difference observed between outdoor infection rate $(4.35 \%, \mathrm{n}=16)$ and indoor infection rate $(4.81 \%, \mathrm{n}=14)$. From the indoor collected mosquitoes, only An. funestus s.l. $(5.20 \%, \mathrm{n}=14)$ were infected while for outdoor collected mosquitoes both An. funestus s.l. $(4.70 \%, \mathrm{n}=1)$ and An. gambiae s.l. $(3.57 \%, \mathrm{n}=2)$ were infected. The infection rate of the specific sibling species is shown in Table 5 .

\section{Discussion}

The present study documents a mortality rate of 75.48 and $78.11 \%$ for deltamethrin and permethrin, respectively in malaria vectors in Kwale County. For the assessment of phenotypic resistance, WHO classifies a population into three categories based on their percentage mortality or susceptibility: A population with 
Table 5 Plasmodium falciparum sporozoite infection rate of Anopheles mosquitoes from Kwale, Coastal Kenya

\begin{tabular}{llrl}
\hline Species & Sibling species & Total tested & \% positive \\
\hline An. funestus s.l. & - & 567 & 4.94 \\
& An. funestus s.s. & 413 & 4.84 \\
& An. leesoni & 25 & 4.00 \\
& An. parensis & 20 & 5.00 \\
& An. rivulorum & 10 & 0 \\
& An. vaneedeni & 4 & 0 \\
& Hybrids & 6 & 33.33 \\
& Not amplified & 89 & 3.74 \\
An. gambiae s.l. & - & 75 & 2.60 \\
& An. arabiensis & 55 & 3.51 \\
& An. gambiae s.s. & 2 & 0 \\
An. coustani & Not amplified & 18 & 0 \\
An. pharoensis & - & 5 & 0 \\
An. squamosus & - & 5 & 0 \\
An. pretoriensis & - & 4 & 0 \\
\hline
\end{tabular}

$100-98 \%$ mortality is regarded susceptible, $97-90 \%$ mortality indicates possible resistance that needs confirmation either using more bioassays or assessing the level of resistant genes while $<90 \%$ indicates resistance [24]. Based on this classification, this study reveals presence of phenotypic resistance to pyrethroids in An. arabiensis, possible resistance in An. gambiae s.s. and susceptibility in An. funestus s.l. In comparison to an earlier study in the Kenyan Coast [32] that showed low level of resistance (83-93\%) to deltamethrin in An. gambiae s.l., this study shows high levels of resistance (62.38\%). This increased resistance levels might be as a result of selection pressure due to increased ITN coverage. However, the contribution of agricultural insecticides should not be ignored.

Though high levels of phenotypic resistance were exhibited, the levels of $k d r$ allele frequency were very low (1.33\%). Surprisingly, while An. arabiensis was more resistant to deltamethrin and permethrin compared to An. gambiae s.s. no $k d r$ mutation was detected in $A n$. arabiensis suggesting that other mechanisms could be contributing to the resistance phenotype observed.

For the species composition of malaria vectors this study shows higher densities of An. funestus s.l. in comparison to An. gambiae s.l. This is in line with previous studies that have reported changes in species composition with relative increase in An. funestus s.l. compared to An. gambiae s.l. These changes have been alluded to insecticide pressure arising from the up-scaling of LTNs/ LLINs and IRS [13, 33, 34].

Although the overall density of An. funestus s.l. was higher than that of An. gambiae s.l, the proportion of
An. funestus s.l. reared from field collected larvae was low compared to the proportion of An. gambiae s.l. This could have occurred as result of high mortality rate for the An. funestus larvae in the insectary during rearing due to the difficulty associated with rearing An. funestus [35].

For the sibling species composition of An. gambiae complex, our study reports An. arabiensis as the dominant sub-species. Previously, in the Kenyan Coast and other regions in Kenya An. gambiae s.s. was the dominant subspecies while An. arabiensis was regarded as a secondary vector [18]. However, since the up-scaling of vector control a reverse in the trends has been reported with a relative increase in $A n$. arabiensis which is regarded as a more opportunistic species relative to $A n$. gambiae s.s. [13, 15].

For An. funestus sibling species composition, this study reveals a more complex composition compared to previous studies in the area where only three subspecies were identified [36]. The present study identifies five sibling species: An. funestus s.s., An. leesoni, An. parensis, An. rivulorum, An. vaneedeni and six hybrids. An. funestus s.s. dominated the An. funestus population with a proportion of $76.02 \%$. This correlates with findings from other studies in the Kenyan Coast and other regions in Kenya where An. funestus s.s. has been reported as the dominant An. funestus sibling species [36-38]. Unlike in the An. gambiae complex, where more exophagic and exophilic species have taken over, this study shows that An. funestus s.s. which is regarded as highly anthropophilic and endophagic remains the dominant sub-species. There is little information on the historical composition of An. funestus sub-species in the Kenyan Coast. In most of the previous studies, An. funestus s.l. has only been identified morphologically, thus it is difficult to tell if there has been any changes in sub-species composition over time. However, the increased complexity reported in this study compared to the two previous studies [36, 38] could be an indicator of possible changes in species composition as a result of the current vector control strategies. However, this could change with season and needs to be evaluated for different seasons and over different years before and after introduction of insecticide-treated bed nets.

In the current study, a higher proportion of malaria vectors was collected outdoor compared to indoor. This is consistent with other studies that have reported increased proportions of malaria vectors outdoor alluded to increased ITN use [39]. While change in species dominance has been linked to increased outdoor proportions, this might be the case for An. gambiae s.l. due to the increase in An. arabiensis. For An. funestus s.l., the dominancy of An. funestus s.s which is more endophagic could 
suggest a possible change in feeding and resting behaviours as a way to avoid insecticides. With the main vector control methods; LLINs and IRS targeting endophilic, endophagic and anthropophilic vectors, both changes in species composition and behavior adjustment pose a big threat to malaria control.

Contrary to recent studies in the area the current study reports a high overall $P$. falciparum infection rate of $4.55 \%[14,19]$. There are several plausible explanations for the high infection rate. First, increased insecticidal interventions over time might have led to reduced susceptibility of malaria vectors to insecticides used to treat nets. This means the nets become less effective in repelling, deterring and killing malaria vectors. Reduced efficacy of bed nets translates to increased human vector contact leading to high infection rate in mosquitoes [40]. Second, differences in sampling season could lead to differences in infection rates. Higher infection rates have been reported in drier seasons compared to wet seasons [13]. For this study mosquitoes were collected during the dry season, July to August. Third, the difference in sampling method could lead to differences in infection rates. This study used light traps and Prokopack aspirator. Light traps have been reported to increase the proportion of infected mosquitoes 2-3 times fold [41, 42]. However, the observed high infection rate might also be as a result of the changing malaria prevalence in the Kenyan Coast. Both previous studies were conducted at a time (20092011) when malaria prevalence was on the fall while the present study was conducted at a time when there are reports of a rising malaria prevalence in the Kenyan Coast [43].

Results from this study show that the rate of $P$. falciparum infection outdoor was the same as that of indoor. This is consistent with other studies that have reported increased $P$. falciparum infected mosquitoes outdoor with increased bed net coverage [38]. The source of outdoor sporozoite infection remains elusive since it is still not known whether the outdoor infection is as a result of the vectors resting outdoors after an infected indoor blood meal or as a result of an infected outdoor blood meal. The latter will have dire consequences on malaria control for the reason that the current vector control methods do not control outdoor biting vectors.

The 100\% insecticide susceptibility exhibited by $A n$. funestus yet they showed the highest level of Plasmodium infection seems counterintuitive. It is possible that An. funestus acquires the infection outdoor thus having reduced contact with insecticide treated nets. However, the possibility of insecticide resistance compromising vector competence should not be ignored as this could also be a plausible explanation for the low P. falciparum infection in An. gambiae.

\section{Conclusions}

Taken together, results from this study report occurrence of insecticide resistance in malaria vectors in the Kenyan Coast. The presence of a low frequency of the L1014S allele in a population exhibiting phenotypic resistance calls for investigation of not only other modes of resistance but also other putative genetic markers of insecticide resistance. The occurrence of high proportions of malaria vectors outdoor highlights the need to augment ITNs and IRS with vector control methods targeting outdoor vectors.

\begin{abstract}
Abbreviations
CDC: Centre for disease control and prevention; ELISA: enzyme linked immunosorbent assay; IRS: indoor residual spraying; KEMRI: Kenya Medical Research Institute; LLINs: long-lasting insecticidal nets; NMCP: National Malaria Control Programme; PCR: polymerase chain reaction; s.l.: sensu lato; s.s.: sensu stricto; WHO: World Health Organization.
\end{abstract}

\section{Authors' contributions}

DMM conceived the idea and designed the study. CWK conducted the sample collection and laboratory analysis and wrote the manuscript with active contribution from other authors. JMM was responsible for the field logistics and supervised the entomological surveys. LK, FAO and WRM offered general supervision of the study. All authors read and approved the final manuscript.

\section{Author details}

${ }^{1}$ School of Biological Sciences, University of Nairobi, P.O. Box 30197, Nairobi 00100 , Kenya. ${ }^{2}$ Department of Biological Sciences, Pwani University, Kilif, Kenya. ${ }^{3}$ Man \& Well-Being Research Office, Science for Health, P.O. Box 44970, Nairobi 00100 , Kenya. ${ }^{4}$ KEMRI-Centre for Geographic Medicine Research, Kilif, Kenya. ${ }^{5}$ KEMRI-Centre for Biotechnology Research and Development, Nairobi, Kenya.

\section{Acknowledgements}

This study was supported by Kenya Medical Research Institute, Internal Research Grant (KEMRI/IRG/135/5). The authors would like to thank Dr. Francis Mutuku for field Logistics support, technical and field staff from Vector-Borne Disease Control Unit (VBDCU) laboratories at Msambweni County Referral Hospital for assistance with sample collection, molecular entomology staff, Centre for Biotechnology Research and Development (CBRD), KEMRI, for assistance with laboratory analysis and the residents of Kidomaya and Marigiza for cooperation during entomological surveys. This paper is published with the permission of the director of Kenya Medical Research Institute.

\section{Competing interests}

The authors declare that they have no competing interests.

\section{Availability of data and materials}

All data generated and used to make conclusions for this study has been included in the article.

\section{Consent for publication}

Not applicable.

\section{Disclaimer}

The findings and conclusions in this report are those of the authors and do not necessarily represent the official position of Kenya Medical Research Institute.

\section{Ethics approval and consent to participate}

The study was approved by KEMRI Scientific Ethical Review Committee (SERU), KEMRI/SERU/CBRD/134/3085 and informed oral consent was obtained from household owners before mosquito collection. 


\section{Funding}

This study was funded by KEMRI Internal Research Grant (KEMRI/IRG/135/5) awarded to Dr. Damaris Matoke-Muhia. The grant facilitated field activities, laboratory sample processing, data analysis and administrative support.

\section{Publisher's Note}

Springer Nature remains neutral with regard to jurisdictional claims in published maps and institutional affiliations.

Received: 7 September 2017 Accepted: 23 December 2017

Published online: 05 January 2018

\section{References}

1. RBM. The global malaria action plan for a malaria free world. Geneva: Roll Back Malaria Partnership; 2008

2. National Malaria Control Programme (NMCP), Kenya National Bureau of Statistics (KNBS), ICF International. Kenya malaria indicator survey 2015. Nairobi: Ministry of Health; 2016.

3. WHO. World malaria report 2014. Geneva: World Health Organization; 2014.

4. RBM. Malaria control today; current WHO recommendations. Geneva: Roll Back Malaria Partnership; 2005.

5. White MT, Conteh L, Cibulskis R, Ghani AC. Costs and cost-effectiveness of malaria control interventions-a systematic review. Malar J. 2011;10:1.

6. WHOPES. Pesticides and their application for the control of vectors and pests of public health importance. 6th ed. Geneva: World Health Organization Pesticide Evaluation Scheme; 2006.

7. Noor AM, Amin AA, Akhwale WS, Snow RW. Increasing coverage and decreasing inequity in insecticide-treated bed net use among rural Kenyan children. PLoS Med. 2007:4:e255.

8. Zhou G, Li JS, Ototo EN, Atieli HE, Githeko AK, Yan G. Evaluation of universal coverage of insecticide-treated nets in western Kenya: field surveys. Malar J. 2014;13:351.

9. IR Mapper. http://www.irmapper.com/. Accessed 12 Nov 2015.

10. Ranson H, N'Guessan R, Lines J, Moiroux N, Nkuni Z, Corbel V. Pyrethroid resistance in African anopheline mosquitoes: what are the implications for malaria control? Trends Parasitol. 2011;27:91-8.

11. Ochomo E, Bayoh NM, Kamau L, Atieli F, Vulule J, Ouma C, et al. Pyrethroid susceptibility of malaria vectors in four districts of Western Kenya. Parasites Vectors. 2014;7:310.

12. Yewhalaw D, Kweka EJ. Insecticide resistance in East Africa-history, distribution and drawbacks on malaria vectors and disease control. In: Trdan S, editor. Agricultural and Biological Sciences. Insecticides resistance. Rijeka: Intech; 2016. p. 189-215.

13. Mwangangi JM, Mbogo CM, Orindi BO, Muturi EJ, Midega JT, Nzovu J, et al. Shifts in malaria vector species composition and transmission dynamics along the Kenyan coast over the past 20 years. Malar J. 2013;12:13.

14. Mutuku FM, King CH, Mungai P, Mbogo C, Mwangangi J, Muchiri EM, et al. Impact of insecticide-treated bed nets on malaria transmission indices on the south coast of Kenya. Malar J. 2011;10:356.

15. Bayoh MN, Mathias DK, Odiere MR, Mutuku FM, Kamau L, Gimnig JE, et al. Anopheles gambiae: historical population decline associated with regional distribution of insecticide-treated bed nets in western Nyanza Province, Kenya. Malar J. 2010;9:62.

16. Reid MC, McKenzie FE. The contribution of agricultural insecticide use to increasing insecticide resistance in African malaria vectors. Malar J. 2016;15:107.

17. Mutuku FM, Khambira M, Bisanzio D, Mungai P, Mwanzo I, Muchiri EM, et al. Physical condition and maintenance of mosquito bed nets in Kwale County, coastal Kenya. Malar J. 2013;12:46.

18. Mbogo CM, Mwangangi JM, Nzovu J, Gu W, Yan G, Gunter JT, et al. Spatial and temporal heterogeneity of Anopheles mosquitoes and Plasmodium falciparum transmission along the Kenyan coast. Am J Trop Med Hyg. 2003;68:734-42.

19. Onyango SA, Kitron U, Mungai P, Muchiri EM, Kokwaro E, King CH, et al. Monitoring malaria vector control interventions: effectiveness of five different adult mosquito sampling methods. J Med Entomol. 2013;50:1140-51.
20. Bøgh C, Pedersen EM, Mukoko DA, Ouma JH. Permethrin-impregnated bednet effects on resting and feeding behaviour of lymphatic filariasis vector mosquitoes in Kenya. Med Vet Entomol. 1998;12:52-9.

21. Mboera LE. Sampling techniques for adult Afrotropical malaria vectors and their reliability in the estimation of entomological inoculation rates. Tanzan Health Res Bull. 2005;7:117-24.

22. Morgan JC. Methods in Anopheles research. Atlanta: Malaria Research and Reference Reagent Resource Center (MR4); 2010.

23. WHO. Test procedures for insecticide resistance monitoring in malaria vectors, bio-efficacy and persistence of insecticides on treated surfaces: report of the WHO informal consultation. Geneva: World Health Organization; 1998.

24. WHO. Test procedures for insecticide resistance monitoring in malaria vector mosquitoes. Geneva: World Health Organization; 2013.

25. Gillies T, Coetzee M. Supplement of the Anopheles of Africa south of Sahara (Afrotropical region). Johannesburg: Publication of the South African Institute of Medical Research; 1987

26. Collins FH, Mendez MA, Rasmussen MO, Mehaffey PC, Besansky NJ, Finnerty V. A ribosomal RNA gene probe differentiates member species of the Anopheles gambiae complex. Am J Trop Med Hyg. 1987;37:37-41.

27. Scott JA, Brogdon WG, Collins FH. Identification of single specimens of the Anopheles gambiae complex by the polymerase chain reaction. Am J Trop Med Hyg. 1993;49:520-9.

28. Koekemoer L, Kamau L, Hunt R, Coetzee M. A cocktail polymerase chain reaction assay to identify members of the Anopheles funestus (Diptera: Culicidae) group. Am J Trop Med Hyg. 2002;66:804-11.

29. Della Torre A, Tu Z, Petrarca V. On the distribution and genetic differentiation of Anopheles gambiae s.s. molecular forms. Insect Biochem Mol Biol. 2005:35:755-69.

30. Bass C, Nikou D, Donnelly MJ, Williamson MS, Ranson H, Ball A, et al. Detection of knockdown resistance $(k d r)$ mutations in Anopheles gambiae: a comparison of two new high-throughput assays with existing methods. Malar J. 2007:6:111.

31. Wirtz RA, Burkot TR. Detection of malarial parasites in mosquitoes. Adv Dis Vector Res. 1991:8:77-106.

32. Msami JE. Monitoring insecticide resistance among malaria vectors in Coastal Kenya. 2013. http://erepository.uonbi.ac.ke:8080/xmlui/handle/123456789/52382. Accessed 16 Nov 2015

33. McCann RS, Ochomo E, Bayoh MN, Vulule JM, Hamel MJ, Gimnig JE, et al. Reemergence of Anopheles funestus as a vector of Plasmodium falciparum in western Kenya after long-term implementation of insecticide-treated bed nets. Am J Trop Med Hyg. 2014;90:597-604.

34. Lwetoijera DW, Harris C, Kiware SS, Dongus S, Devine GJ, McCall PJ, et al. Increasing role of Anopheles funestus and Anopheles arabiensis in malaria transmission in the Kilombero Valley, Tanzania. Malar J. 2014;13:331.

35. Dia I, Guelbeogo MW, Ayala D. Advances and perspectives in the study of the malaria mosquito Anopheles funestus. In: Manguin S, editor. Anopheles mosquitoes - new insights into malaria vectors. Rijeka: InTech Publ; 2013.

36. Kamau L, Munyekenye GO, Koekemoer LL, Hunt RH, Coetzee M. A survey of the Anopheles funestus (Diptera: Culicidae) group of mosquitoes from 10 sites in Kenya with special emphasis on population genetic structure based on chromosomal inversion karyotypes. J Med Entomol. 2003;40:664-71.

37. Kweka E, Kamau L, Munga S, Lee M, Githeko A, Yan G. A first report of Anopheles funestus sibling species in western Kenya highlands. Acta Trop. 2013;128:158-61.

38. Kamau L, Hunt R, Coetzee M. Analysis of the population structure of Anopheles funestus (Diptera: Culicidae) from western and coastal Kenya using paracentric chromosomal inversion frequencies. J Med Entomol. 2002:39:78-83

39. Russell TL, Govella NJ, Azizi S, Drakeley CJ, Kachur SP, Killeen GF. Increased proportions of outdoor feeding among residual malaria vector populations following increased use of insecticide-treated nets in rural Tanzania. Malar J. 2011;10:80

40. Gimnig JE, Vulule JM, Lo TO Kamau L, Kolczak MS, Phillips-Howard PA et al. Impact of permethrin-treated bed nets on entomologic indices in an area of intense year-round malaria transmission. Am J Trop Med Hyg. 2003;68:16-22. 
41. Mbogo CN, Glass GE, Forster D, Kabiru EW, Githure Jl, Ouma JH, Beier JC. Evaluation of light traps for sampling anopheline mosquitoes in Kilif, Kenya. J Am Mosq Control Assoc. 1993;9:260-3.

42. Davis JR, Hall T, Chee EM, Majala A, Minjas J, Shiff CJ. Comparison of sampling of anopheline mosquitoes by light trap and human-bait collections indoor in Bagamoyo, Tanzania. Med Vet Entomol. 1995;9:249-55.
43. Snow RW, Kibuchi E, Karuri SW, Sang G, Gitonga CW, Mwandawiro C, et al. Changing malaria prevalence on the Kenyan Coast since 1974: climate, drugs and vector control. PLoS ONE. 2015;10:e0128792.

\section{Submit your next manuscript to BioMed Central and we will help you at every step:}

- We accept pre-submission inquiries

- Our selector tool helps you to find the most relevant journal

- We provide round the clock customer support

- Convenient online submission

- Thorough peer review

- Inclusion in PubMed and all major indexing services

- Maximum visibility for your research

Submit your manuscript at

www.biomedcentral.com/submit 